\title{
AVALIAÇÃO DAS PROPRIEDADES DE UM COMPÓSITO DE MATRIZ POLIMÉRICA DE POLIPROPILENO RECICLADO REFORÇADO COM RESÍDUO DE MADEIRA
}

\author{
Denise Scheeren ${ }^{1}$, Cláudia Mendes Mählmann², Franciélli Müller ${ }^{2}$ \\ ${ }^{I}$ Curso de Química, Universidade de Santa Cruz do Sul, 96815-900, Santa Cruz do Sul, Brasil. E-mail: \\ denisescheeren@hotmail.com.br \\ ${ }^{2}$ Departamento de Química e Física, Universidade de Santa Cruz do Sul, 96815-900, Santa Cruz do Sul, Brasil. E-mail: \\ mclaudia@unisc.br \\ franciellimuller@unisc.br
}

\section{RESUMO}

Produtos plásticos são ideais para um modelo de consumo rápido e barato, por outro lado, o seu uso momentâneo gera demasiado descarte de resíduos e pode ocasionar problemas ambientais. Neste contexto, o trabalho objetivou produzir um compósito polimérico baseado em materiais pós-consumo, envolvendo polipropileno e farinha de madeira, bem como, avaliar as propriedades das formulações desenvolvidas. Através da reciclagem mecânica de polipropileno (PP) pós-consumo (resíduo urbano) e da farinha de madeira (resíduo do processamento de Medium Density Fiberboard - MDF) proveniente da indústria moveleira, desenvolveu-se um compósito polimérico, com diferentes teores dos constituintes em suas formulações, mediante processos de injeção e moldagem por compressão a quente. As propriedades dos compósitos obtidos foram avaliadas através da realização de ensaios físicos e mecânicos de granulometria, espessura, resistência à tração e flexão, absorção de água e índice de fluidez e morfológico - microscopia eletrônica de varredura (MEV). Através dos resultados obtidos, foi possível destacar que a incorporação da farinha de madeira ao compósito, proporcionou ao material o aumento da resistência à flexão e o aumento da rigidez, entretanto, a resistência à tração diminuiu. $\mathrm{O}$ índice de absorção de água foi maior no compósito que continha maior proporção de farinha de madeira em sua formulação. Já as micrografias evidenciaram a adesão das fibras à matriz e a formação de bolhas nas imagens de seção transversal.

Palavras-chave: Compósito. Farinha de madeira. Medium Density Fiberboard. Polipropileno. Reciclado.

\section{Introdução}

A crescente demanda de utilização de materiais poliméricos, em virtude da sua praticidade e baixo custo, acarreta impactos ambientais por consequência do seu uso indiscriminado e descarte inapropriado, uma vez que sua decomposição pode levar centenas de anos. A responsabilidade compartilhada pelo ciclo de vida dos produtos vem direcionando a cadeia produtiva a desenvolver materiais ambientalmente corretos, a partir do aproveitamento de resíduos sólidos, incentivando a produção e o consumo de produtos derivados de materiais reciclados e recicláveis.

O polipropileno (PP) é um dos plásticos commodities de maior crescimento de consumo, pois apresenta estrutura molecular versátil [1]. Associado ao grande consumo está o grande volume desse termoplástico gerado como resíduo, sendo interessante a reutilização ou reciclagem deste material [2-4], uma das possibilidades de reutilização deste, é utilizá-lo como matriz polimérica de compósitos, que podem ser reforçados com diferentes tipos de materiais [5-6], como o caso da madeira, em forma de fibras, aparas, pó, entre outros [7-8]. Resíduos de madeira podem ter dois principais destinos: matéria-prima para produtos de maior valor agregado (PMVA), ou para fins energéticos (carvão, cabos, briquete, etc.) [9].

O Medium Density Fiberboard (MDF), ou placa de fibra de média densidade é um material compósito à base de madeira (com 10\% de ureia-formaldeído), destinado à montagem de móveis e para a produção de molduras arquitetônicas e para as indústrias de construção civil [10-12]. Na etapa de corte, entre outras, são gerados resíduos na quantidade de $18-20 \%$ em massa de MDF, dentre os quais mais de $90 \%$ estão na forma de serragem fina, com uma faixa de tamanho de partícula entre $10 \mathrm{e}$ $850 \mu \mathrm{m}$ [11]. Ocorre mundialmente um aumento expressivo na produção e consumo de painéis a base de madeira, como o MDF, 
formados por processo de prensagem a seco, madeira desfibrada com adição de resina sintética [12].

Estudos sobre a utilização de diferentes proporções de materiais poliméricos e resíduos de madeira com ou sem adição de compatibilizante [13-14], despertam interesse visto que apresenta inúmeras vantagens, como resistência à umidade e deterioração ambiental, extrusão em perfis com diferentes formatos, resistência ao empenamento e trincas, baixo custo de manutenção, durabilidade em ambientes agressivos tais como marinas e piscinas e ganho ambiental [15-16]. Sidharta [17] descreveu em sua tese as vantagens e desvantagens do WPC (Wood Plastic Composite), se torna alternativa às madeiras maciças. Cresce o número de empresas que oferecem uma linha de madeira ecológica WPC, o produto também é fabricado no Brasil e se apresenta com o conceito de madeira sustentável.

Neste contexto, o objetivo deste trabalho foi produzir compósito polimérico baseado em materiais pós-consumo, envolvendo polipropileno e farinha de madeira, bem como avaliar as propriedades das formulações desenvolvidas.

\section{Metodologia}

\subsection{Matérias-primas}

As matérias-primas utilizadas para o desenvolvimento do compósito polimérico foram: farinha de madeira e polipropileno pós-consumo. A farinha de madeira proveniente da serragem e recortes de chapas e painéis de MDF, fornecida por uma indústria moveleira e o polipropileno proveniente de sobras de potes e tampas de sorvete. Os materiais foram cedidos por indústrias da região do Vale do Taquari (RS - Brasil).

\subsection{Procedimento}

A farinha de madeira foi seca em estufa (Quimis), à $60^{\circ} \mathrm{C}$, até atingir massa constante, posteriormente foi realizada a classificação granulométrica de $2.550 \mathrm{~g}$ (massa total) em um conjunto de peneiras da série Tyler (32, 42, 60 e 80 mesh) com suporte/agitação mecânica, durante um período de 10 minutos. Para o desenvolvimento do compósito foi utilizado o material passante na peneira de 80 mesh, com granulometria menor que $180 \mu \mathrm{m}$.

O polipropileno foi previamente fragmentado em moinho de facas MECANOFAR MF 300, no qual foram obtidos flakes, que foram lavados em água corrente com detergente neutro e posteriormente secos em estufa à $70^{\circ} \mathrm{C}$ por $8 \mathrm{~h}$.

As matérias primas foram homogeneizadas de acordo com as formulações indicadas na Tabela 1, em homogeneizador de laboratório ( $\mathrm{MH}$ equipamentos), este material foi posteriormente moído em moinho de facas (MECANOFAR MF 300), e processado de acordo com os seguintes métodos:

a) por injeção em injetora HIMACO, com temperatura de $215^{\circ} \mathrm{C}$, obtendo os corpos de prova e,

b) por moldagem por compressão a quente, em temperatura de $180^{\circ} \mathrm{C}, 20$ toneladas de fechamento, em prensa de placas paralelas - MH equipamentos, obtendo placas com dimensões de 400x400 mm e espessura de $4 \mathrm{~mm}$ e posterior recorte dos corpos de prova.

As formulações 100PP, 95PP5FM e 85PP15FM foram processadas de acordo com os métodos (a) e (b). A formulação 75PP25FM foi processada apenas pelo método (b).

\begin{tabular}{lccl}
\multicolumn{5}{c}{ Tabela 1 - Formulações e método de processamento } & \multicolumn{1}{c}{ Método } \\
\hline \multicolumn{1}{c}{ Formulação } & PP $(\boldsymbol{\%} \mathbf{~ m})$ & $\mathbf{F M ~} \mathbf{( \%} \mathbf{~ m})$ & \\
\hline 100PP-I & 100 & 0 & Injeção \\
95PP5FM-I & 95 & 5 & Injeção \\
85PP15FM-I & 85 & 15 & Injeção \\
100PP-C & 100 & 0 & Moldagem por compressão \\
95PP5FM-C & 95 & 5 & Moldagem por compressão \\
85PP15FM-C & 85 & 15 & Moldagem por compressão \\
75PP25FM-C & 75 & 25 & Moldagem por compressão \\
\hline
\end{tabular}

Não foi possível realizar a injeção da formulação 75PP25FM pela formação de gases já observada na formulação 85PP15FM durante a injeção, necessitando constantes interrupções do processo, fato também observado por Yamaji, F. M. \& Bonduelle [18-19], que descrevem que a madeira quando processada em temperaturas de até $150^{\circ} \mathrm{C}$ libera vapor d'água, em temperaturas na faixa de 150 e $200^{\circ} \mathrm{C}$ ocorrem reações químicas que favorecem a formação de gases e o escurecimento da madeira.

\subsection{Avaliação de Propriedades}

\subsubsection{Espessura}

A determinação de espessura dos corpos de prova foi realizada com o uso de micrômetro digital com resolução de 0,001 mm, marca Mitutoyo. Foram realizadas três medidas (centro e extremidades) em cada corpo de prova e realizado o cálculo da média.

\subsubsection{Resistência à flexão}

Os ensaios de flexão foram realizados de acordo com a norma ASTM D790 [20], realizados na máquina universal de 
ensaios (EMIC DL 10.000), com a determinação das propriedades de resistência à flexão, módulo de elasticidade e deformação sob flexão, propriedades estas, importantes para o controle de qualidade e para avaliação do desempenho dos materiais plásticos.

\subsubsection{Resistência à tração}

Os ensaios de resistência mecânica por tração seguiram o procedimento descrito na norma ASTM D638 [21], com célula de carga $50 \mathrm{kgf}$, velocidade $10 \mathrm{~mm} \cdot \mathrm{min}^{-1}$. Foram avaliados os parâmetros de deformação específica, módulo de elasticidade e tensão na força máxima. Ensaio realizado com o uso de equipamento universal de ensaios mecânicos (EMIC DL 10.000).

\subsubsection{Absorção de água}

O ensaio foi realizado pelo método de gravimetria, em triplicata, de acordo com a norma ASTM D570 [22]. Amostras dos corpos de prova medindo $1 \mathrm{~cm}^{2}$ cada, foram secas em estufa por 24 horas, sob temperatura de $50^{\circ} \mathrm{C} \pm 1^{\circ} \mathrm{C}$ e posteriormente pesadas. Em seguida, as amostras foram imersas em água deionizada e pesadas individualmente em intervalos de 15 minutos até atingir peso constante. A quantidade de água absorvida foi determinada conforme Equação 1.

$$
\text { Absorção de água }=\underline{\text { M úmida }- \text { M seca }} \times 100
$$

$$
\text { M seca }
$$

\subsection{5 Índice de fluidez}

O teste foi desenvolvido em um plastômetro descrito no método ASTM D1238 [23], foi utilizado o equipamento Melt Flow Index Junior CEAST. A temperatura utilizada no ensaio foi de $190^{\circ} \mathrm{C}$ com carga de $2,16 \mathrm{~kg}$.

\subsection{Ensaio morfológico}

\subsubsection{Microscopia eletrônica de varredura (MEV)}

Foram recortadas amostras de $0,5 \mathrm{~cm}^{2}$ dos corpos de prova fraturados no ensaio de tração, das formulações 100PP, 95PP5FM, 85PP15FM e 75PP25FM, amostras foram previamente metalizadas com ouro para posterior análise de superfície e seção transversal, em microscópio eletrônico de varredura Philips modelo XL 20.

\section{Resultados e discussões}

\subsection{Granulometria}

Na Tabela 2 observa-se a distribuição do tamanho das partículas da farinha de madeira, que variou entre $500 \mu \mathrm{m}$ e menor que $180 \mu \mathrm{m}$.

\begin{tabular}{cccccc}
\multicolumn{6}{c}{ Tabela 2 - Classificação granulométrica da farinha de madeira. } \\
\hline $\begin{array}{c}\text { Malha } \\
\text { mesh }\end{array}$ & $\mathbf{\mu m}$ & $\begin{array}{c}\text { Massa } \\
\text { Retida } \\
(\mathbf{g})\end{array}$ & $\begin{array}{c}\text { \% Retida } \\
\text { Individual } \\
(\mathbf{m})\end{array}$ & $\begin{array}{c}\text { \% Retida } \\
\text { Acumulada }\end{array}$ & $\begin{array}{c}\% \\
\text { Passante }\end{array}$ \\
\hline 32 & 500 & 276 & 10,92 & 10,92 & 89,08 \\
42 & 355 & 422 & 16,69 & 27,61 & 72,39 \\
60 & 250 & 564 & 22,31 & 49,92 & 50,08 \\
80 & 180 & 318 & 12,58 & 62,50 & 37,5 \\
Fundo & $<180$ & 948 & 37,5 & 100,00 & 0 \\
\hline
\end{tabular}

O equivalente a 37,5\% do material ficou retido no fundo, seguido pela peneira de 60 mesh com mais de $22 \%$ de material retido. O gráfico da Figura 1 apresenta os resultados de percentual de massa de farinha de madeira retida nas diferentes peneiras usadas.

O resíduo da farinha de madeira possui uma distribuição por tamanho de partículas bastante heterogênea.

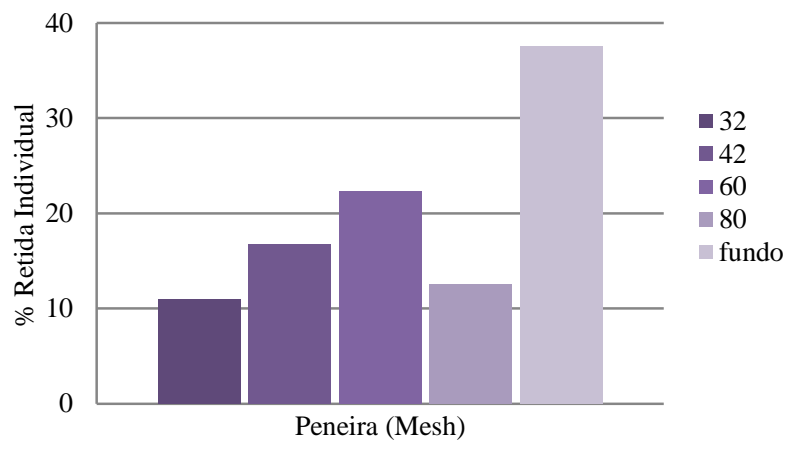

Figura 1 - Resultados da porcentagem retida individualmente por peneira. Fonte: o autor.

Verificou-se que mais de $70 \%$ do material ficou retido entre as peneiras 60 e $<80$ mesh (fundo), sendo que $22,31 \%$ (m) ficaram retidos na peneira de 60 mesh e $37,5 \%$ (m) no fundo.

A partir destes resultados fez-se a escolha do tamanho da partícula a ser utilizada na formulação do compósito, que foi < $80 \mathrm{mesh}=<180 \mu \mathrm{m}-$ material retido no fundo, com o intuito de atingir melhor homogeneidade entre fibra e matriz e facilitar o processo de injeção.

\subsection{Espessura}

$\mathrm{Na}$ Tabela 3 são apresentados os valores médios de espessura dos corpos de prova avaliados. 
Tabela 3 - Valores médios de espessura $(\mathrm{mm})$ dos corpos de prova dos ensaios de flexão e tração.

\begin{tabular}{lcc}
\hline \multicolumn{1}{c}{ Formulação } & $\begin{array}{c}\text { Ensaio de Flexão } \\
\text { Espessura (mm) }\end{array}$ & $\begin{array}{c}\text { Ensaio de Tração } \\
\text { Espessura (mm) }\end{array}$ \\
\hline 100PP-I & 3,25 & 3,07 \\
95PP5FM-I & 3,26 & 3,09 \\
85PP15FM-I & 3,26 & 3,07 \\
100PP-C & 3,63 & 3,49 \\
95PP5FM-C & 4,34 & 4,62 \\
85PP15FM-C & 4,42 & 4,67 \\
75PP25FM-C & 4,12 & 4,10 \\
\hline
\end{tabular}

Os corpos de prova injetados apresentaram espessura superior a $3 \mathrm{~mm}$, enquanto os corpos de prova obtidos a partir das placas obtidas por moldagem por compressão foram superiores a $4 \mathrm{~mm}$.

\subsection{Absorção de água}

Na Figura 2 são apresentados os resultados do ensaio de absorção de água. O tempo de análise foi determinado até atingimento de massa constante ou redução.

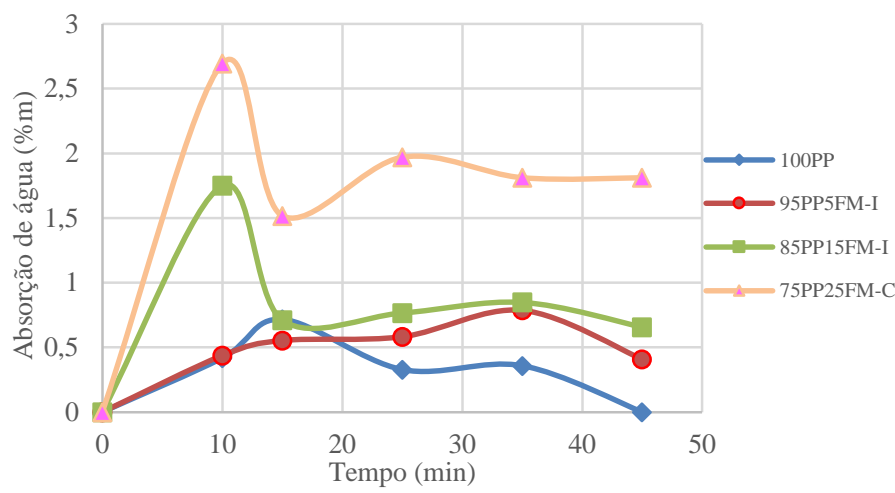

Figura 2 - Resultados do percentual de absorção de água. Fonte: o autor.

As amostras 85PP15FM-I e 75PP25FM-C foram as que absorveram maior porcentagem de água nos 10 minutos iniciais, logo após, notou-se um decréscimo na absorção de água das mesmas e novamente uma crescente aos 25 minutos de análise. A partir dos 35 minutos, a formulação 75PP25FM-C manteve massa constante e as demais tiveram redução.

Quanto maior a quantidade de madeira na formulação, maior a absorção de água. Resultado esperado, pois em sua estrutura interna os corpos de prova apresentaram bolhas, que facilitaram a penetração da água no material, e visto que a madeira tem característica hidrofílica. A variação do teor de umidade pode provocar uma rede de micro fraturas na superfície do compósito, que pode alterar suas propriedades mecânicas [24].

\section{4 Índice de fluidez}

Na Figura 3 são apresentados os resultados alcançados através da determinação do índice de fluidez das formulações estudadas. Para este ensaio, a título de comparação de resultados, foi incluída a amostra de polipropileno puro (potes e tampas moídos - 100PP) antes de passar pelo processo de mistura.

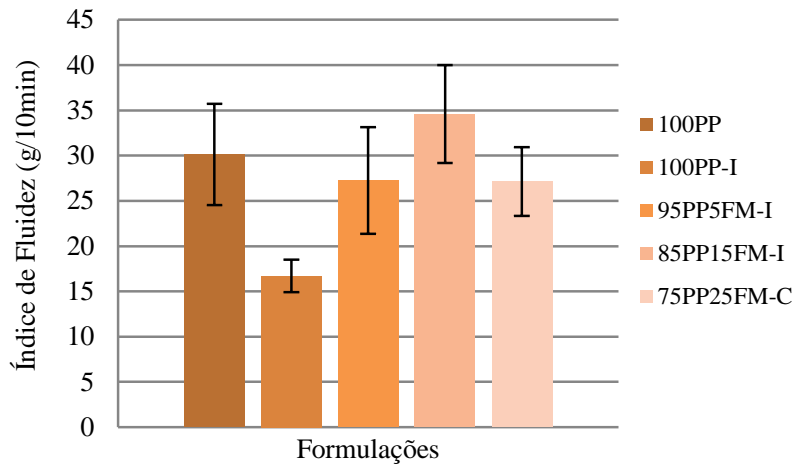

Figura 3 - Resultados de índice de fluidez. Fonte: o autor.

Observa-se que não houve variação significativa do índice de fluidez entre as formulações 100PP (antes da mistura), 95PP5FM-I, 85PP15FM-I, 75PP25FM-C (30,14 g/10 min; 27,25 $\mathrm{g} / 10 \mathrm{~min} ; 34,58 \mathrm{~g} / 10 \mathrm{~min}$ e $27,13 \mathrm{~g} / 10 \mathrm{~min}$, respectivamente). A amostra 100PP-I apresentou índice mais baixo $(16,71 \mathrm{~g} / 10 \mathrm{~min})$. Battistelle, R. et al. [25] encontraram valor muito próximo (30 $\mathrm{g} / 10 \mathrm{~min}$ ), para a amostra controle 100PP, e concluíram em seu estudo, que com o aumento da proporção de farinha de madeira ocorre a diminuição do índice de fluidez.

\subsection{Resistência à flexão}

Para que um material obtenha um bom desempenho mecânico, deve haver uma boa adesão entre as fases da fibra e da matriz. Na tabela 4 são apresentados os resultados obtidos para o ensaio em flexão e em tração. 


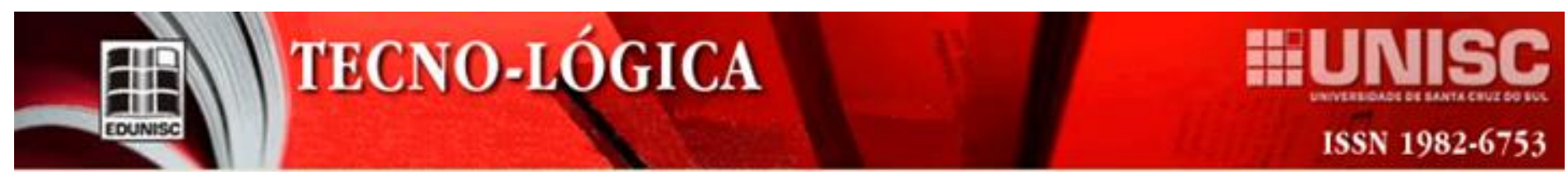

Tabela 4 - Resultados dos ensaios mecânicos de flexão e tração e do desvio padrão das formulações estudadas.

\begin{tabular}{|c|c|c|c|c|c|c|}
\hline \multirow[b]{2}{*}{ Formulação } & \multicolumn{3}{|c|}{ Ensaio de Flexão } & \multicolumn{3}{|c|}{ Ensaio de Tração } \\
\hline & $\begin{array}{c}\text { Módulo de } \\
\text { Elasticidade (MPa) } \\
\end{array}$ & $\begin{array}{c}\text { Tensão } \\
\text { Máxima (MPa) } \\
\end{array}$ & $\begin{array}{c}\text { Def. Específica } \\
\text { Máxima (\%) }\end{array}$ & $\begin{array}{c}\text { Módulo de } \\
\text { Elasticidade (MPa) } \\
\end{array}$ & $\begin{array}{c}\text { Tensão Força } \\
\text { Máxima (MPa) }\end{array}$ & $\begin{array}{c}\text { Def. Força } \\
\text { Máxima (mm) }\end{array}$ \\
\hline 100PP-I & $912,80 \pm 61,80$ & $21,67 \pm 2,01$ & $4,44 \pm 0,23$ & $595,00 \pm 137,60$ & $21,59 \pm 0,98$ & $11,60 \pm 0,87$ \\
\hline 95PP5FM-I & $542,50 \pm 53,30$ & $24,90 \pm 0,51$ & $4,43 \pm 0,15$ & $250,80 \pm 219,50$ & $20,21 \pm 0,39$ & $13,48 \pm 1,16$ \\
\hline 85PP15FM-I & $796,40 \pm 58,49$ & $27,11 \pm 1,35$ & $4,00 \pm 0,29$ & $889,70 \pm 40,51$ & $17,45 \pm 0,49$ & $8,36 \pm 0,21$ \\
\hline 100PP-C & $1467,00 \pm 106,60$ & $24,47 \pm 1,38$ & $2,87 \pm 0,13$ & $823,00 \pm 16,09$ & $12,99 \pm 0,78$ & $5,99 \pm 0,43$ \\
\hline 95PP5FM-C & $1120,00 \pm 428,20$ & $25,45 \pm 7,97$ & $2,57 \pm 0,41$ & $759,50 \pm 33,51$ & $10,59 \pm 1,51$ & $5,53 \pm 0,57$ \\
\hline 85PP15FM-C & $1417,00 \pm 78,41$ & $22,05 \pm 1,19$ & $3,06 \pm 0,50$ & $807,30 \pm 54,05$ & $11,93 \pm 1,18$ & $6,01 \pm 0,30$ \\
\hline 75PP25FM-C & $459,20 \pm 433,40$ & $11,56 \pm 1,33$ & $1,54 \pm 0,15$ & $715,20 \pm 92,34$ & $4,21 \pm 1,02$ & $4,38 \pm 0,15$ \\
\hline
\end{tabular}

Os resultados do ensaio de flexão para o parâmetro módulo de elasticidade são apresentados na Figura 4.

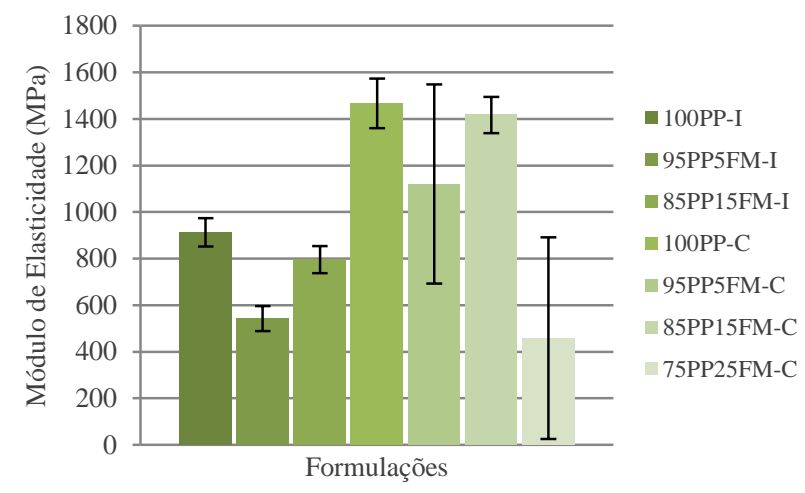

Figura 4 - Resultados do módulo de elasticidade. Fonte: o autor.

As formulações que apresentaram maior valor de módulo de elasticidade foram 85PP15FM-C (1417,00 MPa) seguido por 95PP5FM (1120,00 MPa). As formulações com maior módulo têm características de material mais rígido. Hillig, E. et al. [26] obtiveram resultados próximos aos encontrados, 1509,40 MPa para o compósito de formulação HDPE com adição de $20 \%$ de MDF. No estudo de Battistelli, R. et al. [25], a formulação $90 \%$ PP com adição de $10 \%$ FM apresentou módulo de elasticidade de $1390 \mathrm{MPa}$.

Os resultados do ensaio de flexão, para tensão na força máxima são apresentados na Figura 5.

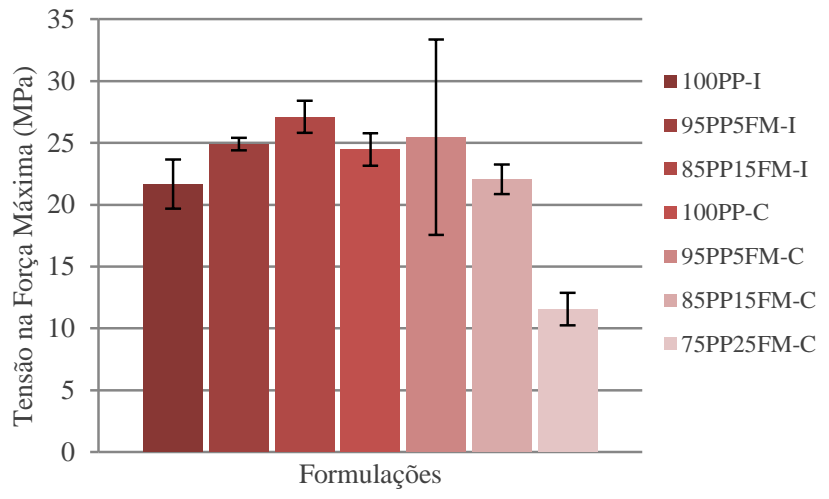

Figura 5 - Resultados de tensão na força máxima. Fonte: o autor.

Verificou-se que a proporção 85PP15FM-I apresentou maior tensão na força máxima. Esta mistura, resultou em um valor médio de resistência à flexão de $27,11 \mathrm{MPa}$ com desvio padrão de $\pm 1,35$, conforme Tabela 4 .

A formulação 95PP5FM-C superaria este resultado se somado seu desvio padrão máximo, resultando em 33,42 MPa de tensão. Resultado que fica próximo ao encontrado por Hillig, E. et al. [26] de 36,61 MPa para a formulação HDPE + $20 \%$ de serragem. Apenas a formulação 75PP25FM-C obteve resistência à tração inferior ao 100PP-I.

Constatou-se que para os corpos de prova injetados o aumento da proporção de farinha de madeira melhorou a resistência do material. 
Os resultados do ensaio de flexão, para o parâmetro deformação específica máxima, são apresentados na Figura 6.

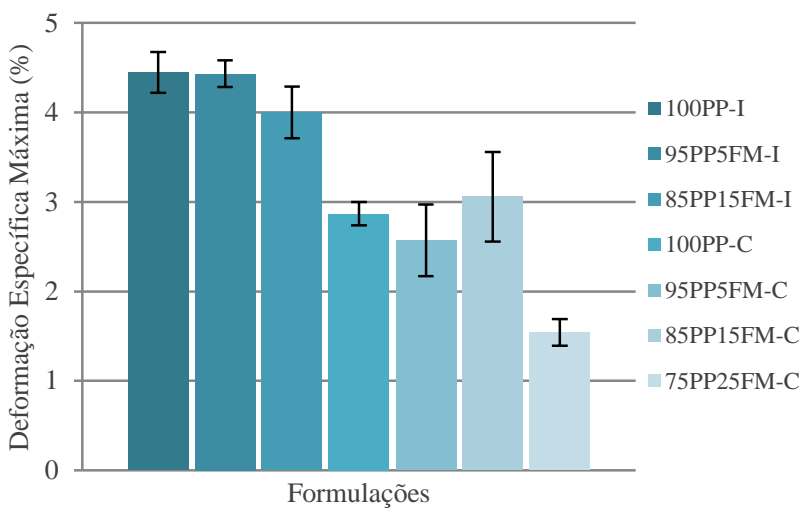

Figura 6 - Resultados do percentual de deformação específica máxima. Fonte: o autor.

Observou-se que quanto maior a quantidade de farinha de madeira nas formulações, menor é a sua deformação específica.

As formulações 95PP5FM-I e 85PP15FM-I obtiveram resultados próximos à amostra 100PP-I, bem como as formulações 95PP5FM-C e 85PP15FM-C da amostra 100PP-C.

\subsection{Resistência à tração}

De acordo com Canevarolo [27], o módulo de elasticidade está diretamente relacionado com a rigidez do polímero, ou seja, quanto maior for o valor do módulo, maior sua rigidez.

Na Figura 7 são apresentados os resultados do ensaio de tração para o parâmetro módulo de elasticidade.

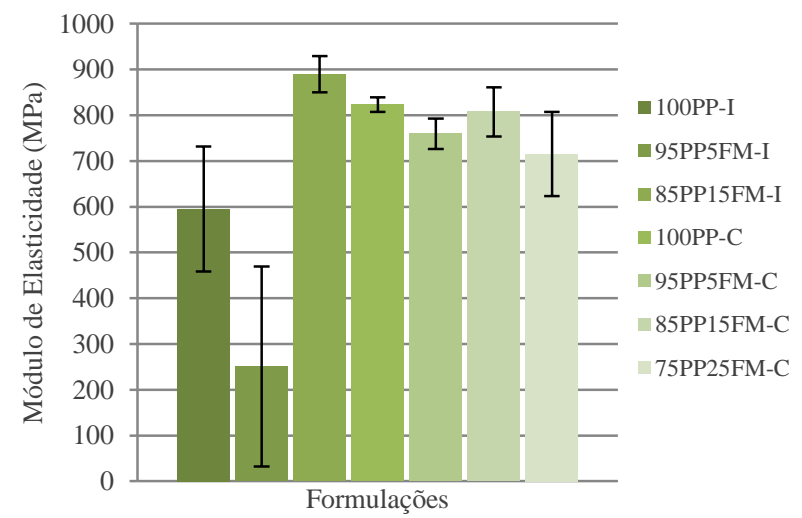

Figura 7 - Resultados do módulo de elasticidade. Fonte: o autor.

O valor do módulo de elasticidade para o PP injetado (100PP-I) foi de $595 \mathrm{MPa}$ e por moldagem por compressão (100PP-C) foi de $823 \mathrm{MPa}$.
A formulação que apresentou maior módulo foi 85PP15FM-I. As formulações produzidas por ambos os processos superaram as propriedades da amostra controle (100PP-I). Apenas a mistura 95PP5FM-I apresentou módulo inferior, conforme Tabela 4 e Figura 7.

Em seus estudos, Grison et al. [28], realizaram testes mecânicos em compósito encontrando valores como 699,90 MPa para o módulo de elasticidade para a formulação PEAD com adição de $14 \%$ madeira e $2 \%$ de compatibilizante, e 507,90 $\mathrm{MPa}$ para o PEAD puro, valor que se aproxima dos resultados encontrados neste estudo para 100PP-I (595,00 MPa).

Os resultados de tensão na força máxima do ensaio de tração são apresentados na Figura 8.

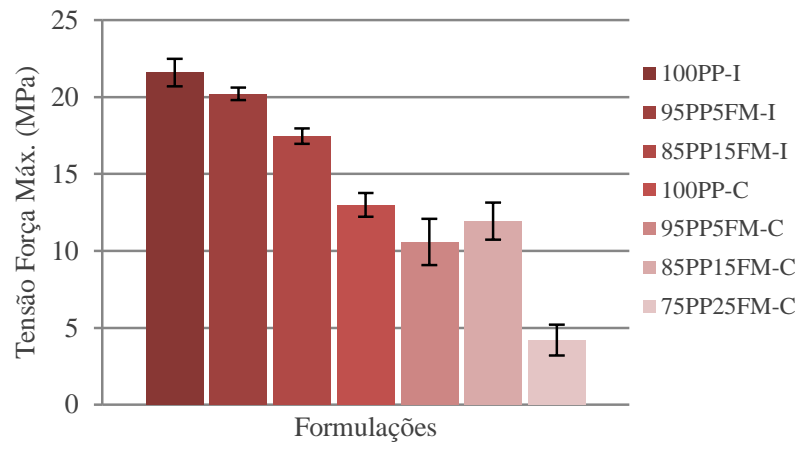

Figura 8 - Resultados de tensão na força máxima. Fonte: o autor.

Verificou-se que os corpos de prova 100PP-I apresentaram o maior valor de tensão na força máxima e também foi possível observar que quanto maior a proporção de farinha de madeira adicionada à formulação do compósito, menor sua resistência à tração. Apenas a formulação 85PP15FM-C superou a resistência à tração da amostra 100PP-C. A formulação 95PP5FM-I apresentou valor de resistência à tração muito próxima ao 100PP-I.

Yamaji [18] também observou que com o aumento da proporção de madeira em compósitos com PP, ocorre a diminuição da resistência à tração. Hillig, E. et al. [26] encontraram valores médios de resistência à tração para o compósito HDPE com adição de $20 \%$ de MDF igual a 28,35 $\mathrm{MPa}$. 


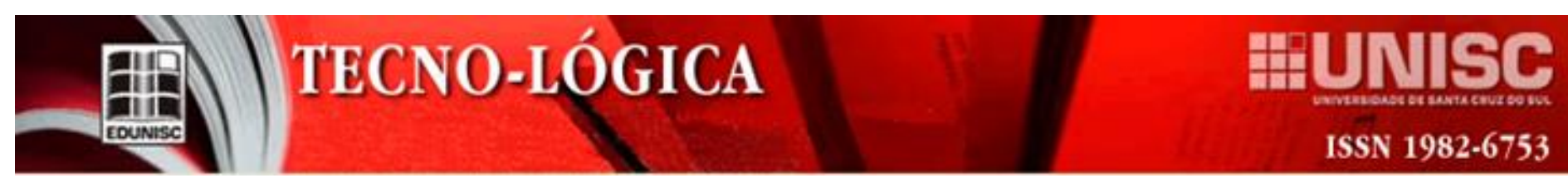

Na Figura 9 são apresentados os resultados da deformação na força máxima para o ensaio de tração.

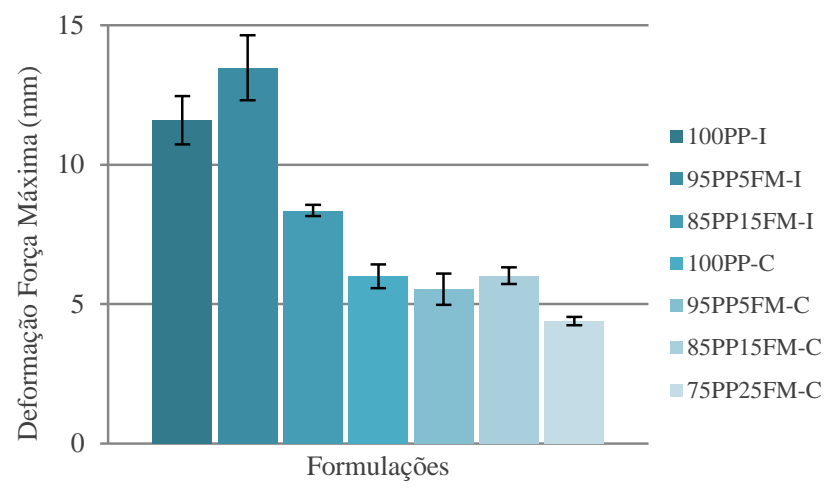

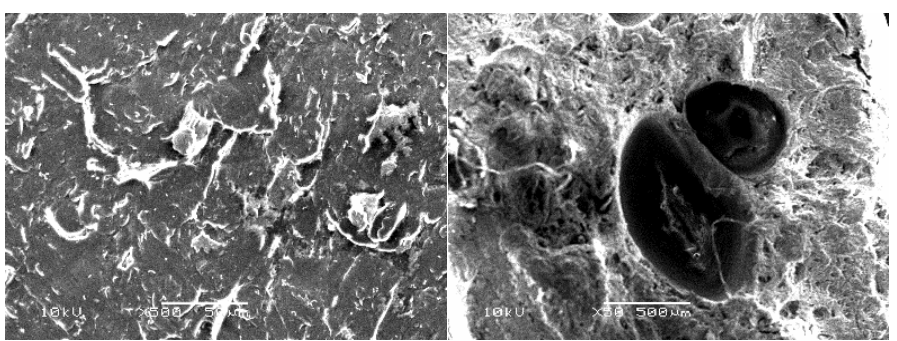

(a)

Figura 11- Micrografia de superfície da formulação 95PP5FM-I com aumento de 500x (a) e Micrografia de seção transversal da formulação 95PP5FM-I com aumento de 50x (b).

A partir da micrografia de superfície da Figura 11(a) foi possível observar que as fibras se encontravam aderidas a matriz, porém, quando analisamos a seção transversal 11(b), verificou-se que ocorre a formação de bolhas.

Figura 9 - Resultados de deformação na força máxima. Fonte: o autor.

A formulação que apresentou maior deformação na força máxima, superior ao 100PP-I foi a 95PP5FM-I com 13,48 mm. Em contraponto ao comportamento da mistura 75PP25FM-C que deformou apenas $4,38 \mathrm{~mm}$.

Esse desempenho inferior dos compósitos com maior proporção de madeira, também foi observado por Yamaji [18] e pode ser justificado pela maior dificuldade de se obter uma mistura homogênea, com o aumento do percentual de madeira na mistura.

\subsection{Microscopia Eletrônica de Varredura}

A interface entre fibra e matriz influencia as propriedades de reforço dos compósitos, determinando a capacidade de transferir as tensões da matriz para a fibra e a resistência do material compósito.

Nas Figuras 10 a 15 são apresentadas as micrografias de superfície e seção transversal das formulações estudadas.

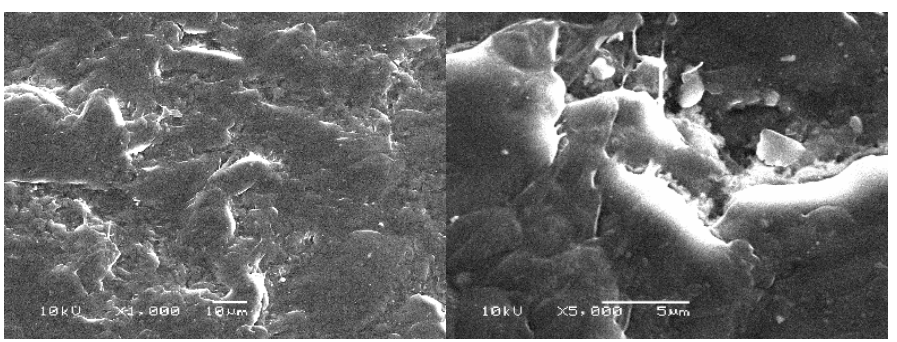

(a)

Figura 10 - Micrografia de superfície da formulação 100PP-I com aumento de 1000x (a) e Micrografia de superfície da formulação 100PP-I com aumento de $5000 x(b)$.

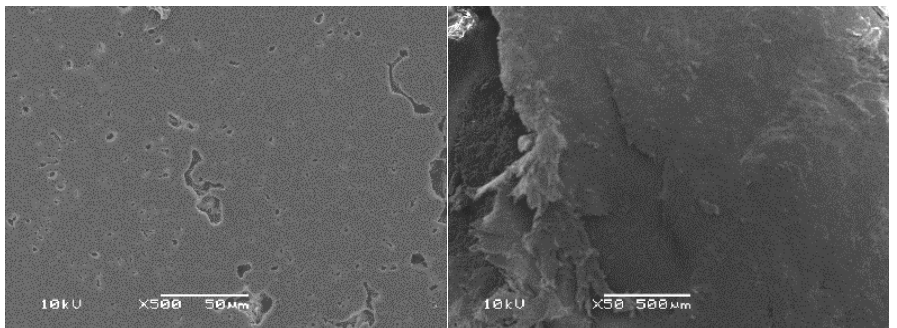

(a)

(b)

Figura 12 - Micrografia de superfície da formulação 95PP5FM-C com aumento de 500x (a) e Micrografia de seção transversal da formulação 95PP5FM-C com aumento de 50x (b).

Nas micrografias da formulação 95PP5FM-C foi possível visualizar que houve melhor aderência entre a fibra e a matriz, e consequentemente o material pode ser considerado mais resistente.

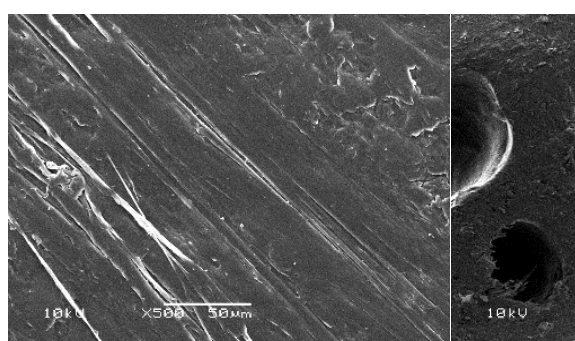

(a)

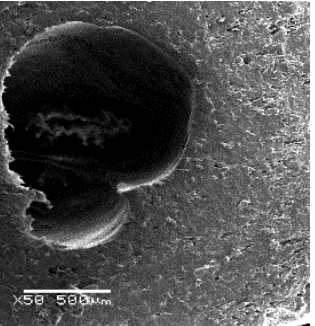

(b)
Figura 13 - Micrografia de superfície da formulação 85PP15FM-I com aumento de 500x (a) e Micrografia de seção transversal da formulação 85PP15FM-I com aumento de 50x (b)

A formulação 85PP15FM-I apresentou comportamento semelhante à formulação 95PP5FM-I, sendo que na Figura 13(b) também pode ser observado o surgimento de bolhas. 


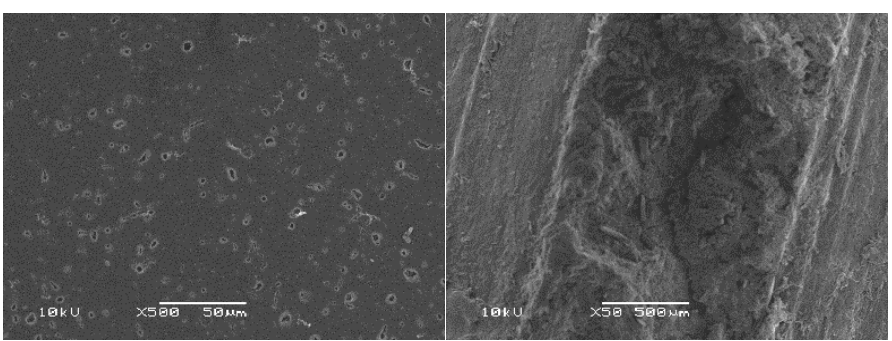

(a)

(b)

Figura 14 - Micrografia de superfície da formulação 85PP15FM-C com aumento de 500x (a) e Micrografia de seção transversal da formulação 85PP15FM-C com aumento de 50x (b).

$\mathrm{Na}$ Figura 14(a) e (b) foi possível destacar que a formulação 85PP15FM-C apresentou boa aderência entre a fibra e a matriz, e consequentemente o material pode ser considerado mais resistente.

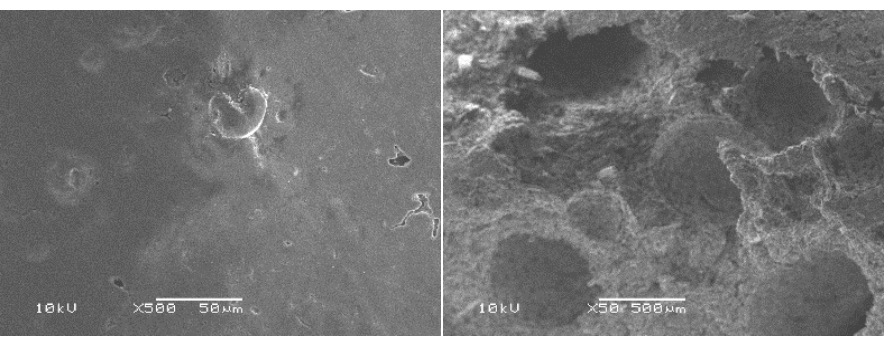

(a)

(b)

Figura 15 - Micrografia de superfície da formulação 75PP25FM-C com aumento de 500x (a) e Micrografia de seção transversal da formulação 75PP25FM-C com aumento de 50x (b).

$\mathrm{Na}$ Figura 15(b) pode ser novamente observado a formação de bolhas. Este comportamento é consequência da liberação de gases durante o aquecimento do material, em decorrência do processo de fusão do plástico com a madeira, a umidade contida na mistura transformou-se em vapor, que foi eliminado juntamente com o material durante o processo de injeção e moldagem por compressão a quente.

\section{Conclusões}

Com base nos resultados obtidos através dos ensaios mecânicos, foi possível destacar que as formulações do polipropileno reciclado com reforço de resíduo de farinha de madeira (MDF), proporcionaram ao material o aumento da resistência à flexão.

Além disso, verificou-se que a adição de farinha de madeira favoreceu a rigidez do material. Porém, com o aumento da proporção de farinha de madeira ao compósito houve a diminuição da resistência à tração. Logo, a resistência à tração não influenciou na melhoria das características do compósito.
O ensaio morfológico possibilitou evidenciar que diversas formulações apresentaram a formação de bolhas em sua estrutura interna, resultante da liberação de umidade durante o aquecimento da mistura, entretanto, houve interação entre fibra e matriz, com destaque para as formulações 95PP5FM-C e 85PP15FM-C

Os resultados obtidos indicam que a formulação de compósito polimérico baseado em materiais pós-consumo é possível.

\section{Agradecimentos}

Os autores agradecem o apoio técnico recebido pelos funcionários e bolsistas dos laboratórios de ensino e pesquisa dos Departamentos de Química e Física e Engenharia Ambiental da Universidade de Santa Cruz do Sul, bem como aos técnicos do Laboratório de Materiais Poliméricos do Centro de Microscopia e Microanálise da Universidade Federal do Rio Grande do Sul.

Às empresas Sorvebom e Movelas que gentilmente cederam as matérias-primas para o desenvolvimento deste compósito polimérico.

\section{EVALUATION OF THE PROPERTIES OF A POLYPROPYLENE COMPOSITES OF RECYCLED POLYPROPYLENE REINFORCED WITH WOOD RESIDUE.}

ABSTRACT: Plastic products are ideal for a cheap and fast consumption model, on the other hand, their momentary use generates too much waste disposal and can cause environmental problems. In this context, this work aimed to produce a polymeric composite based on post-consumer materials, involving polypropylene and wood flour, as well as to evaluate the properties of the formulations developed. Through the mechanical recycling of polypropylene (PP) (urban waste) and wood flour (processing waste of Medium Density Fiberboard $\mathrm{MDF}$ ) from the furniture industry, a polymeric composite was developed, with different constituent contents in formulations, through injection processes and hot compression molding. The properties of the composites obtained were evaluated through physical and mechanical granulometry tests, thickness, tensile and flexural strength, water absorption and fluidity and morphological index - scanning electron microscopy (MEV). Through the obtained results, it was possible to highlight that the incorporation of wood flour into the composite, provided the material with increased flexural strength and increased stiffness, however, the tensile strength decreased. The water absorption index was higher in the composite that had higher proportion of wood flour in its formulation. And the micrographs showed the 
adhesion of the fibers to the matrix and the bubbles formation in the transversal section images.

Keywords: Composite. Wood flour. Medium Density Fiberboard. Polypropylene. Recycled.

\section{Referências}

[1] MANRICH, Silvio. Processamento de Termoplásticos. São Paulo: Artliber Editora, 2005

[2] M. Xanthos. Recycling of the \#5 polymer. Science, 337 (6095) (2012), p. 700.

[3] N. Singh et al. Recycling of plastic solid waste: a state of art review and future applications. Compos. B Eng., 115 (2017), pp. 409-422. https://doi.org/10.1016/j.compositesb.2016.09.013.

[4] Jenna R. Jambeck et al. Plastic waste inputs from land into the ocean. Science, 347 (6223) (2015), pp. 768-771. DOI: 10.1126/science.1260352.

[5] Jmal H. et al. Influence of the grade on the variability of the mechanical properties of polypropylene waste. Waste Manag. 75 (2018), pp. 160-173.

[6] Brachet, P. et al. Modification of mechanical properties of recycled polypropylene from post-consumer containers. Waste Manag., 28 (12) (2008), pp. 2456-2464.

[7] K.L., Pickering; M.G. Aruan Efendy; T.M. Le; A review of recent developments in natural fiber composites and their mechanical performance; Composites Part A: Applied Science and Manufacturing. Volume 83, Pages 98112,2016

[8] Martinez, Lopez Y. et al. Production of wood-plastic composites using cedrela odorata sawdust waste and recycled thermoplastics mixture from post-consumer products - A sustainable approach for cleaner production in Cuba. Journal of Cleaner Production. Volume 244, 20 January 2020, 118723.

[9] Ministério do Meio Ambiente. Secretaria de Mudanças Climáticas e qualidade ambiental. Secretaria de biodiversidade e florestas. Projeto PNUD BRA 00/20 - Apoio as políticas públicas na área de gestão e controle ambiental. Aproveitamento de resíduos e subprodutos florestais, alternativas tecnológicas e propostas de políticas ao uso de resíduos florestais para fins energéticos Sumário executivo - Revisão 00. Novembro de 2009. Curitiba-PR. Disponível em:

<http://www.mma.gov.br/estruturas/164/_publicacao/164_publicacao100120110 33501.pdf> Acesso em: 03 de nov. 2018.

[10] COVOLATO, R.A. Desenvolvimento de compósito constituído de madeira e resina ureia formaldeído com cura incentivada por micro-ondas. Dissertação (mestrado) apresentada à Escola de Engenharia Mauá do Centro Universitário do Instituto Mauá de Tecnologia. São Caetano do Sul, 2007. Disponível em: $\langle$ https://maua.br/biblioteca/consultas/dissertacoes/processos-quimicos $>$. Acesso em: 15 de nov. 2018.

[11] GAN, Q.; ALLEN, S.J.; MATTHEW, R. Activation of waste MDF sawdust charcoal and its reactive dye adsorption characteristics. Waste Management, Belfast, vol. 24, pág. 841-848, fev. 2004. Disponível em: <https://doi.org/10.1016/j.wasman.2004.02.010>. Acesso em: out. de 2018.

[12] ELEOTÉRIO, Jakson R. Propriedades físicas e mecânicas de painéis MDF de diferentes densidades e teores de resinas. Dissertação (Mestrado em Materiais e Processos de Fabricação) - Universidade de São Paulo, fev. 2000. Disponível em: 〈http://coral.ufsm.br/cienciaflorestal/artigos/v10n2/art6v10n2.pdf〉. Acesso em: nov. 2018.
[13] LISPERGUER, Justo; et al. Efecto de las características de harina de madera en las propiedades físico-mecánicas y térmicas de polipropileno reciclado. Maderas, Cienc. tecnol. vol.15 n $n^{\circ} .3$ Concepción oct. 2013 Epub 24Jul-2013. Disponível em: <https://scielo.conicyt.cl/scielo.php?script= sci_arttext\&pid=S0718-221X2013000300006 >. Acesso em: 03 nov. 2018.

[14] FERNANDES Beatriz L., DOMINGUES, Antonio J. Caracterização mecânica de polipropileno reciclado para a indústria automotiva. Polímeros, v.17 n.2 São Carlos: abr./jun. 2007. Disponível em: <http://www.scielo.br/pdf/po/v17n2/a05v17n2.pdf>. Acesso em: out. de 2018.

[15] CORREA, C. A. et al. Compósitos Termoplásticos com Madeira. Polímeros: Ciência e Tecnologia, vol. 13, $\mathrm{n}^{\circ}$ 3, p. 154-165, 2003. Disponível em: <http://www.scielo.br/pdf/po/v13n3/v13n3a05.pdf〉. Acesso em: set. de 2018.

[16] CHANHOUN, Marius; et al. Study of the implementation of waste wood, plastics and polystyrenes for various applications in the building industry Elsevier, Construction and Building Materials. 167 (2018) 936-941. Disponível em: <https://doi.org/10.1016/j.conbuildmat.2018.02.080>. Acesso em: set. de 2018.

[17] SIDHARTA, Andre. Wood Plastic Composites (WPCs) as an Alternative to Solid Lumber - The University of British Columbia, Vancouver: British Columbia, 2011. Disponível em: < https://open.library.ubc.ca/cIRcle/collections/undergraduateresearch/52966/items /1.0103128>. Acesso em: out. de 2018 .

[18] YAMAJI, FÁBIO MINORU. Produção de compósito de plástico- madeira a partir de resíduos da indústria madeireira. Tese apresentada como requisito parcial à obtenção do grau de Doutor em Ciências Florestais, Programa de PósGraduação em Engenharia Florestal, Setor de Ciências Agrárias, Universidade Federal do Paraná, 2004. Disponível em: < https://acervodigital.ufpr.br/bitstream/handle/1884/819/Yamaji;jsessionid=CD91 AA98FFF2C342A806CC29E7CCBB49?sequence=1>. Acesso em: out. de 2018.

[19] Yamaji, F.M e Bonduelle A. Utilização da serragem na produção de plástico-madeira. Revista Floresta 34 (1), Jan/Abr 2004, 59-66, Curitiba-PR. Disponível em: <https://revistas.ufpr.br/floresta/article/viewFile/2375/1984>. Acesso em: nov. de 2018

[20] AMERICAN SOCIETY FOR TESTING AND MATERIALS. ASTM D790 - Standard Test Methods for Flexural Properties of Unreinforced and Reinforced Plastics and Electrical Insulating Materials.

[21] AMERICAN SOCIETY FOR TESTING AND MATERIALS. ASTM D638- Standard Test Method for Tensile Properties of Plastics.

[22] AMERICAN SOCIETY FOR TESTING AND MATERIALS. ASTM D570 - Standard Test Method for Water Absorption of Plastics.

[23] AMERICAN SOCIETY FOR TESTING AND MATERIALS. ASTM D1238 - Standard Test Method for Melt Flow Rates of Thermoplastics by Extrusion Plastometer.

[24] RAYMUNDO, E. A. et al. Análise de absorção de água em compósito de polímero reforçado com fibras naturais. Cadernos UniFOA. Edição Especial do Curso de Mestrado Profissional em Materiais - junho/2012. Disponível em: $\langle$ http://revistas.unifoa.edu.br/index.php/cadernos/article/download/1147/1047>. Acesso em: set. de 2018.

[25] BATTISTELLE, R.; VIOLA, N.; BEZERRA, B.;VALARELLI, I. Caracterização física e mecânica de um compósito de polipropileno reciclado e farinha de madeira sem aditivos. Revista Matéria, v.19, n.01, pp. 07-15, 2014. 


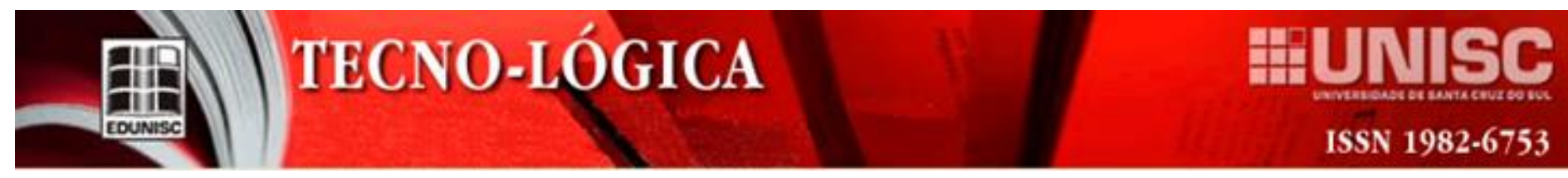

Disponível em: <http://www.scielo.br/pdf/rmat/v19n1/1517-7076-rmat-19-0100007.pdf>. Acesso em: set. de 2018.

[26] HILLIG, E. et al. Caracterização de compósitos produzidos com polietileno de alta densidade (HDPE) e serragem de indústria moveleira - Parte II - Extrusão em dupla rosca. Ciência Florestal, Santa Maria, v. 21, n. 2, p. 335-347, abr-jun, 2011. Disponível em: <http://www.scielo.br/pdf/cflo/v21n2/1980-5098-cflo-2102-00335.pdf>. Acesso em: out. de 2018.

[27] CANEVAROLO, Sebastião V. Jr. Ciência dos polímeros. Um texto básico para tecnólogos e engenheiros. $2^{\mathrm{a}}$ edição. São Paulo: Artliber Editora, 2006.

[28] GRISON, Karine; et al. Avaliação das propriedades mecânicas e morfológicas de compósitos de PEAD com pó de Pinus taeda e alumina calcinada. 2015. Polímeros, 25(4), 408-413, 2015. Disponível em: < http://www.scielo.br/pdf/po/v25n4/0104-1428-po-25-4-408.pdf>. Acesso em: set. de 2018. 